\title{
Color image simulation for underwater optics
}

\author{
Matthieu Boffety ${ }^{1}$, Frédéric Galland ${ }^{1, *}$ and Anne-Gaëlle Allais ${ }^{2}$ \\ ${ }^{1}$ Institut Fresnel, CNRS, Aix-Marseille Université, Ecole Centrale Marseille, Campus de Saint-Jérôme, 13013 \\ Marseille, France \\ ${ }^{2}$ Ifremer, Centre de Méditerranée, Zone portuaire de Brégaillon, 83507 La Seyne-sur-Mer, France \\ *: Corresponding author : Frédéric Galland, email address : frederic.galland@fresnel .fr
}

\begin{abstract}
:
Underwater optical image simulation is a valuable tool for oceanic science, especially for the characterization of image processing techniques such as color restoration. In this context simulating images with a correct color rendering is crucial. This paper presents an extension of existing image simulation models to RGB imaging. The influence of the spectral discretization of the model parameters on the color rendering of the simulated images is studied. It is especially shown that, if only RGB data of the scene chosen for simulations are available, a spectral reconstruction step prior to the simulations improves the image color rendering.
\end{abstract}

Since the pioneering works of the early 1970's [1-4], optical imaging has known a growing interest in the field of oceanic science and engineering [5-7]. However the scattering and absorption properties of water are critical limitations for optical measurements making imaging and system design in subsea environment challenging tasks. Consequently being able to foresee operating conditions is of great interest for a broad range of applications such as experimental planning, performance assessment in turbid environment and image processing characterization in underwater conditions. In this context, being able to simulate realistic underwater images taking into account the whole process of light underwater propagation and measurement appears very valuable. Various approaches have thus been previously developed to simulate underwater image formation $[4,8-10]$, with areas of applications ranging from performance assessment and system design $[4,10]$ to benchmarking of image restoration algorithms [7, 11-13].

Furthermore light propagation in water implies various phenomena that modify the spectral content of an image, such as attenuation or backscattering [14] (i.e. light that is directly 
scattered by the water toward the camera without reaching the scene of interest). Studying color degradation impact on the image quality and on the performance of image processing techniques, such as color restoration algorithms [7,15-19], is thus an important field of underwater image processing. For this purpose, it is therefore crucial to simulate images with a color rendering as realistic as possible. Nevertheless the generalization and characterization of existing simulation methods for underwater color imaging has not been fully addressed yet.

In order to tackle these points, this paper presents a model for simulating underwater RGB images under artificial illumination, which relies on well-known existing approaches. The main purpose of this study is to analyze the evolution of the color rendering of the simulated images according to the discretization of the spectral data used in the formation model. The goal is to highlight the potential difficulties due to RGB image simulation in the context of image processing algorithm characterization, such as color restoration algorithms.

For that purpose, the first section introduces the model underlying the approach in the case where the spectral reflectance of the simulated marine scene is perfectly known. In section 2 , the influence of the number of spectral channels used to simulate underwater color images is then analyzed. Finally in section 3, this study is extended to the case where only RGB data of the scene chosen for the simulations are available instead of multispectral data. It highlights the interest of using a first reconstruction step in order to retrieve additional spectral information about the scene.

\section{The color image formation model}

The approach discussed in this paper relies on Jaffe-McGlamery image formation model [4, 10]. For self-consistency this model is recalled in the following subsection.

\section{A. The "monochromatic" Jaffe-McGlamery approach}

In this article, it is assumed that a given underwater scene is illuminated with an artificial source and observed through an optical sensor (cf. Fig. 1), such as a CCD camera. According to Jaffe-McGlamery approach, the irradiance $\mathcal{E}_{\text {tot }, \lambda}\left(\right.$ in $\left.\mathrm{W} \cdot \mathrm{m}^{-2} \cdot \mathrm{nm}^{-1}\right)$ received by the camera for a given wavelength $\lambda$ and a given point located at $(x, y)$ reads

$$
\mathcal{E}_{\text {tot }, \lambda}(x, y)=\mathcal{E}_{\mathrm{u}, \lambda}(x, y)+\mathcal{E}_{\mathrm{b}, \lambda}(x, y)
$$

with $\mathcal{E}_{\mathrm{b}, \lambda}$ being the contribution due to backscattered light (i.e. light that has been scattered

directly to the camera by the medium without reaching the scene). In Eq. (1), $\mathcal{E}_{\mathrm{u}, \lambda}$ is the total contribution of the scene on the image, defined by

$$
\mathcal{E}_{\mathrm{u}, \lambda}(x, y)=e^{-c_{\lambda} R_{c}} \mathcal{E}_{0, \lambda}(x, y)+\mathcal{E}_{0, \lambda}(x, y) * h_{\lambda}\left(x, y, Z_{o}\right)
$$


where $*$ is the convolution operator over $(x, y), c_{\lambda}$ is the beam total attenuation coefficient of the water, $h_{\lambda}$ is the point spread function (PSF) that accounts for light scattering $[4,8]$, $Z_{o}$ is the distance to the observed scene and $R_{c}$ is the distance between the camera and the observed point $(x, y)$ as defined in Fig 1.(a). In Eq. (2), $\mathcal{E}_{0, \lambda}$ is the irradiance measured by the camera in the absence of water and reads [4]

$$
\mathcal{E}_{0, \lambda}(x, y)=\frac{\pi \mathcal{T}_{\lambda}}{4 N_{l}^{2}}\left(\frac{Z_{o}-F_{l}}{Z_{o}}\right)^{2} \mathcal{L}_{\lambda}(x, y) \cos ^{4} \theta
$$

where $\theta$ is defined in Fig. 1.(a) and where $F_{l}, N_{l}$ and $T_{\lambda}$ are respectively the focal length, f-number and total transmission of the lens. In Eq. (3), $\mathcal{L}_{\lambda}$ is the scene radiance which, for a lambertian scene of reflectance $\mathcal{R}_{\lambda}$, reads $\mathcal{L}_{\lambda}(x, y)=\mathcal{R}_{\lambda}(x, y) \mathcal{E}_{\mathrm{s}, \lambda}(x, y, z=0) / \pi$, with $\mathcal{E}_{\mathrm{s}, \lambda}$ being the received irradiance at location $(x, y, z)$. It is given by

$$
\mathcal{E}_{\mathrm{s}, \lambda}(x, y, z)=e^{-c_{\lambda} R_{s}} \mathcal{E}_{\mathrm{s}, 0, \lambda}(x, y, z)+\mathcal{E}_{\mathrm{s}, 0, \lambda}(x, y, z) * h_{\lambda}\left(x, y, Z_{s}-z\right)
$$

where $\mathcal{E}_{\mathrm{s}, 0, \lambda}$ is the irradiance due to the source and received at the point of coordinates $(x, y, z)$ in the absence of water, $R_{s}$ is the distance between the observed point $(x, y, z)$ and the source (cf. Fig. 1.(a)), and $Z_{s}$ is the distance between the source and the observed plan as defined in Fig. 1.(a).

Various water PSF can be found in literature [20]. All the results shown in this study rely however on the following function $[4,10,21]$

$$
h_{\lambda}(x, y, Z)=\left(e^{-G_{\lambda} Z}-e^{-c_{\lambda} Z}\right) \mathrm{FT}^{-1}\left\{e^{-B_{\lambda} Z \omega}\right\}_{x, y}
$$

where $Z$ is the distance to the considered plan, $G_{\lambda}$ and $B_{\lambda}$ are two empirical factors, $\mathrm{FT}^{-1}$ is the inverse Fourier transform and $\omega$ the angular frequency. In the following, $G_{\lambda}$ and $B_{\lambda}$ have been chosen according to Ref. [21], i.e. $G_{\lambda}=a_{\lambda}$ and $B_{\lambda}=b_{\lambda}$, with $a_{\lambda}$ and $b_{\lambda}$ being respectively the beam absorption and scattering coefficients of water.

For the purpose of the backscattering contribution $\mathcal{E}_{\mathrm{b}, \lambda}$ calculation, the water column is divided into thin slabs, each of them made up of small elementary volumes that scatter the light directly to the camera (cf. Fig 1.(b)). The contribution $\mathcal{E}_{k, \lambda}$ of each volume is calculated using the water volume scattering function $\beta_{\lambda}$, and reads [4]

$$
\mathcal{E}_{\mathrm{k}, \lambda}\left(x, y, z_{k}\right)=\mathcal{E}_{\mathrm{k}, 0, \lambda}\left(x, y, z_{k}\right) e^{-c_{\lambda} R_{k}}+\mathcal{E}_{\mathrm{k}, 0, \lambda}\left(x, y, z_{k}\right) * h_{\lambda}\left(x, y, Z_{k}\right)
$$

with

$$
\mathcal{E}_{k, 0, \lambda}\left(x, y, z_{k}\right)=\frac{\mathcal{T}_{\lambda}}{4 N_{l}^{2}}\left(\frac{Z_{o}-F_{l}}{Z_{o}}\right)^{2} \times \beta_{\lambda}\left(\varphi_{k}\right) \mathcal{E}_{\mathrm{s}, \lambda}\left(x, y, z_{k}\right) \pi \cos ^{3} \theta_{k} \Delta z
$$

where $\Delta z$ is the discretization step of the water column, $\mathcal{E}_{\mathrm{s}, \lambda}\left(x, y, z_{k}\right)$ the illumination that reaches the volume (see Eq. (4)), $R_{k}$ is the distance between the elementary volume and the 
CCD (cf. Fig. 1.(b)) and $Z_{k}=Z_{o}-z_{k}$. Moreover these volumes are generally out of focus and the measured irradiance for a given water slab has therefore to be modified using the defocus point spread function $D_{\text {defocus }}$. According to Ref. [22], $D_{\text {defocus }}$ can be modeled by a Gaussian kernel of parameter $\sigma=\eta d$ where $\eta$ is a constant of proportionality depending on the characteristics of a given camera and $d$ is given by

$$
d(z)=\frac{F_{l}^{2} z}{N\left(Z_{o}-z\right)\left(Z_{o}-F_{l}\right)}
$$

In all the experiments reported in this study it has been chosen $\sigma=d / 2$. Therefore the final expression for the backscattering contribution is [4]

$$
\mathcal{E}_{\mathrm{b}, \lambda}(x, y)=\left.\sum_{k=1}^{Z_{o} / \Delta z}\left[\mathcal{E}_{\mathrm{k}, \lambda} * D_{\text {defocus }}\right]\left(x, y, z_{k}\right)\right|_{z_{k}=\Delta z \times(k-1 / 2)}
$$

The discretization step $\Delta z$ should be chosen small enough to guarantee the convergence of the sum of Eq. (7).

\section{B. $R G B$ color image simulation}

Jaffe-McGlamery image formation model allows the calculation of the measured irradiance for a given wavelength $\lambda$ according to Eq. (1). However, to the best of our knowledge, a detailed study on the simulation of RGB color images in underwater environment has not been addressed yet.

In this section, an approach to simulate RGB images is proposed. It relies on the JaffeMcGlamery "monochromatic" model when taking into account the spectral response of the detector. For that purpose, let $\phi^{c}(\lambda)$ be the detector spectral response in channel

$c \in\{R, G, B\}$. In standard photometry theory, the general form of the signal measured in channel $c$ by a camera [23] can be written as

$$
S_{c}(x, y)=\int_{\lambda} \mathcal{Q}^{c}(\lambda) \phi^{c}(\lambda) \mathcal{E}_{\mathrm{tot}, \lambda}(x, y) \mathrm{d} \lambda
$$

where $\mathcal{Q}^{c}$ is a calibration factor depending on the imaging system characteristics. In this study, for clarity purpose and without any loss of generality, $\mathcal{Q}^{c}(\lambda)=1$ is chosen. In this case the measured signal is thus homogeneous to an irradiance (in $\mathrm{W} \cdot \mathrm{m}^{-2}$ ) and is thus equal to

$$
\mathcal{E}^{c}(x, y)=\int_{\lambda} \phi^{c}(\lambda) \mathcal{E}_{\mathrm{tot}, \lambda}(x, y) \mathrm{d} \lambda
$$

In the case of a spectrum discretized with $N$ wavelengths $\left\{\lambda_{1}, \lambda_{2}, \ldots, \lambda_{N}\right\}$, one obtains

$$
\mathcal{E}_{N}^{c}(x, y) \approx \Delta \lambda \sum_{n=1}^{N} \phi^{c}\left(\lambda_{n}\right) \mathcal{E}_{\mathrm{tot}, \lambda_{n}}(x, y)
$$


where $\Delta \lambda$ is the discretization step, or similarly

$$
\mathcal{E}_{N}^{c}(x, y)=\mathcal{E}_{\mathrm{U}, N}^{c}(x, y)+\mathcal{E}_{\mathrm{B}, N}^{c}(x, y)
$$

with

$$
\begin{aligned}
& \mathcal{E}_{\mathrm{U}, N}^{c}(x, y)=\Delta \lambda \sum_{n=1}^{N} \phi^{c}\left(\lambda_{n}\right) \mathcal{E}_{\mathrm{u}, \lambda_{n}}(x, y) \\
& \mathcal{E}_{\mathrm{B}, N}^{c}(x, y)=\Delta \lambda \sum_{n=1}^{N} \phi^{c}\left(\lambda_{n}\right) \mathcal{E}_{\mathrm{b}, \lambda_{n}}(x, y)
\end{aligned}
$$

From Eq. (8) it is thus possible to simulate realistic underwater RGB images. For that purpose, it is required to take into account not only the water optical properties and the spectral reflectivity of the scene but also the spectral response of the camera. This approach is referred as the direct multispectral approach in the study.

In this paper, two types of water have been considered and their inherent optical properties $a_{\lambda}, b_{\lambda}$ and $c_{\lambda}=a_{\lambda}+b_{\lambda}$ are given in Fig. 2.(a) and (b). Type 1 (resp. type 2) water corresponds to clear (resp. turbid) water. For both water types, the volume scattering function (VSF) $\beta_{\lambda}$ has been taken from Ref. [9] and is given by

$$
\beta_{\lambda}(\varphi)=p(\varphi) b_{\lambda}
$$

where $p(\varphi)$ is shown in Fig. 2.(c). These data were adapted by the author of Ref. [9] from the measurements of Ref. [2] which are VSF standard models [27-30]. The imaging system characteristics used in the study have been chosen according to the set-up of Ref. [9]: The sensor is assumed to be a CCD camera equipped with a lens of focal length $F_{l}=27.5 \mathrm{~mm}$, f-number $N_{l}=2.8$ and transmission $\mathcal{T}_{\lambda}=1$ over the whole spectrum. The observed scene is illuminated by an isotropic punctual white source with unit power output. This source is assumed to be always in the same plane as the camera (i.e. $Z_{o}=Z_{s}$ ) but translated by $1 \mathrm{~m}$ in the $y$ direction (cf. Fig. 1). Moreover two RGB cameras have been simulated. Their spectral responses $\left\{\phi^{R}, \phi^{G}, \phi^{B}\right\}$ are shown in Fig. 3.(a) for camera I and Fig. 3.(b) for camera II. They have been respectively deduced from spectral responses given in Ref. [24] and Ref. [25], and slightly modified in order to have simple shapes, their maxima at the same RGB wavelengths, and the same energy in each channel (i.e. $\sum_{n} \phi^{c}\left(\lambda_{n}\right) \Delta \lambda=1, c \in\{R, G, B\}$ ).

Once specified both the water optical properties and the source/camera characteristics, one can simulate RGB images corresponding to any scene with a known reflectance map $\mathcal{R}_{\lambda}(x, y)$. In this study, the chosen scene is made up of 20 patches whose spectral reflectance has been taken from an existing database [26]. The patches were chosen to have a broad variety of reflectivities over the scene in order to avoid specific data-related behaviors as best 
as possible. Fig. 4 shows the two RGB color images that are obtained when this scene is imaged with both camera types in the absence of water.

As illustration, Fig. 5 shows the results of the simulation for both types of camera and water, in the case of a scene-camera distance $Z_{o}=7 \mathrm{~m}$. All images were simulated using Eq. (8) with $N=100$ wavelengths over a spectral interval ranging from $402 \mathrm{~nm}$ to $798 \mathrm{~nm}$ with a $4 \mathrm{~nm}$ discretization step. It can be seen that, for both water types, the spectral components of the images are strongly affected by the medium but in different ways. Images in type 1 water have a strong blue hue whereas images in type 2 water have a more greenish hue. This is due to an attenuation spectrum flatter in the 400-600nm interval for type 2 water, leading to a rather similar contribution of the green and blue channels on the final image, whereas with type 1 water the minimum of attenuation is clearly in the blue part of the spectrum leading to a strong blue rendering. Moreover the scattering coefficient is almost one order of magnitude higher in type 2 water therefore increasing the contribution of scattered light on the measurement. It results in the presence of a strong veiling light on the image of Fig. 5.(b) and (d), and a severe loss of visibility. It is also obvious that the type of camera impacts the spectral behavior of the image, especially for type 2 water: Image of Fig. 5.(d) being more turquoise than image of Fig. 5.(b). It is therefore mandatory to take into account the spectral response of the camera to simulate realistic underwater RGB images.

\section{Influence of the spectral discretization}

The approach underlying Eq. (8) requires to know all the spectral characteristics of the model (scene reflectance, water optical properties, detector spectral response, ...) for all the considered wavelengths. For example, the previous images of Fig. 5 have been simulated knowing these characteristics for $N=100$ wavelengths. Nevertheless one can wonder whether using such a complete multispectral approach is necessary and if a simpler approach consisting in using fewer wavelengths could not be good enough to obtain realistic images. To answer this question, it is proposed in this section to study the impact of the coarseness of the spectrum discretization on the visual rendering of the backscattering contribution $\mathcal{E}_{\mathrm{B}, N}^{c}$ and of the scene contribution $\mathcal{E}_{\mathrm{U}, N}^{c}$ (defined respectively in Eq. (11) and Eq. (10)).

Let $\mathcal{E}_{\bullet}^{c}$,true be the ideal "ground truth" one should obtain with the image simulator, with $c \in\{R, G, B\}$ and $\bullet \in\{\mathrm{U}, \mathrm{B}\}$. To evaluate quantitatively the quality of the image simulation, the error $\epsilon_{\bullet}^{c}(N)$ between the ground truth image $\mathcal{E}_{\bullet}^{c}$,true and the image simulated with $N$ wavelengths $\mathcal{E}_{\bullet, N}^{c}$ is determined by averaging their absolute difference over the whole pixels of the image:

$$
\epsilon_{\bullet}^{c}(N)=\frac{1}{P} \sum_{x, y}\left|\mathcal{E}_{\bullet, N}^{c}(x, y)-\mathcal{E}_{\bullet, \text { true }}^{c}(x, y)\right|
$$


where $\bullet \in\{\mathrm{U}, \mathrm{B}\}$ and $P$ is the number of pixels in the images. For this analysis, the spectral characteristics of the simulation model parameters are chosen similarly to subsection 1.B (i.e. over a 402-798nm spectral interval with a $4 \mathrm{~nm}$ discretization step), leading to a ground truth spectrum discretization with $N=100$. Therefore $\mathcal{E}_{\bullet \text {,true }}^{c}=\mathcal{E}_{\bullet, N=100}^{c}$, with $c \in\{R, G, B\}$ and $\bullet \in\{\mathrm{U}, \mathrm{B}\}$ while the notation $\mathcal{E}_{\bullet}^{c}, N$ implies that the image is simulated with $N<100$ wavelengths chosen according to a regular sampling.

\section{A. Calculation of the backscattering contribution $\mathcal{E}_{B, N}^{c}$}

Contrary to $\mathcal{E}_{\mathrm{U}, N}^{c}$, the backscattering contribution $\mathcal{E}_{\mathrm{B}, N}^{c}$ is independent of the scene and the two contributions do not necessarily have the same behavior. Therefore, as a first step, only the contribution $\mathcal{E}_{\mathrm{B}, N}^{c}$ is considered.

Fig. 6 shows the evolution of the error $\epsilon_{\mathrm{B}}^{c}$, as a function of the number $N$ of used wavelengths, obtained in each channel for camera I (black dashed curves) and camera II (gray plain curves) in the case of type 1 water. The same configuration as in subsection 1.B is considered with a scene-camera distance $Z_{o}=15 \mathrm{~m}$. Very similar curves are obtained for type 2 water and are thus not shown for clarity. From these curves, it is possible to highlight a general tendency independently of the water type or the considered spectral channel. First of all, errors are more important in the channels that suffer from high water attenuation (mainly the red and green channels for type 1 water). Indeed the spectrum is strongly modified where the attenuation is high, therefore it requires a fine discretization to correctly model the image spectrum degradation. Moreover the precision improves as the number $N$ of wavelengths used for the simulation increases and errors stabilize for a given number of wavelengths, in the studied cases $N \geq 25$ approximately, meaning that $\mathcal{E}_{\mathrm{B}, N}^{c}$ can be simulated with good precision from 25 wavelengths only $\left(\epsilon_{\mathrm{B}}^{c} \leq 1 \%\right.$ for the three channels). A key point to emphasize is that $\mathcal{E}_{\mathrm{B}, N}^{c}$ is independent of the knowledge of the scene characteristics. Therefore, whatever the available scene spectral discretization is, it is always possible to simulate $\mathcal{E}_{\mathrm{B}, N}^{c}$ with a sufficiently fine spectral discretization, provided $a_{\lambda}, b_{\lambda}$ and $c_{\lambda}$ are known for such a discretization.

\section{B. Calculation of the scene contribution $\mathcal{E}_{U, N}^{c}$}

Knowing the spectral reflectance of the scene is generally a key issue of image simulation, since global models of marine scene reflectance are not available contrary to models of water optical properties which can be found for various types of water [30]. The difficulty to obtain realistic scene reflectance data (either by imaging a real scene with a multispectral sensor or by simulating it as in section 1.B) increases with the number of required wavelengths. Therefore it is now proposed to study the influence of the wavelength number on the quality of the simulation of $\mathcal{E}_{\mathrm{U}, N}^{c}$. The goal is to determine the number that has typically to be 
considered for realistic underwater RGB image simulation.

For that purpose, the 20-patch scene of subsection 1.B (cf. Fig. 4) is considered. To study the impact of the reflectance spectral discretization, a reflectance map $\mathcal{R}_{\lambda}^{N}$ is created over $N$ wavelengths $\left\{\lambda_{1}^{(N)}, \lambda_{2}^{(N)}, \cdots, \lambda_{N}^{(N)}\right\}$. For that purpose, starting from the initial 100-wavelength spectral reflectance $\mathcal{R}_{\lambda_{i}}^{100}$ with $i \in[1,100], \mathcal{R}_{\lambda}^{N}$ is directly deduced by averaging $\left\{\mathcal{R}_{\lambda_{i}}^{100}\right\}$ according to the equation

$$
\mathcal{R}_{\lambda_{n}^{(N)}}^{N}(x, y)=\frac{1}{J_{n}} \sum_{j \in \Omega_{n}^{(N)}} \mathcal{R}_{\lambda_{j}}^{100}(x, y)
$$

where $\Omega_{n}^{(N)}$ are subsets containing $J_{n}$ consecutive wavelengths of the full discretization so that $\sum_{n=1}^{N} J_{n}=100$.

Fig. 7 and Fig. 8 show the evolution of the error $\epsilon_{\mathrm{U}}^{c}$ as a function of $N$ for the red channel which corresponds to the channel with the highest relative error (in percentile of the maximum value of the channel $\mathcal{E}_{\mathrm{U} \text {,true }}^{R}$ ). Calculations were performed for both types of camera (black dashed lines and gray plain lines) in type 1 water for $Z_{o}=15 \mathrm{~m}$ (Fig. 7 ) and in type 2 water for $Z_{o}=7 \mathrm{~m}$ (Fig. 8). From these curves it can be seen that errors in the red channel are higher for camera II than camera I. This can be explained by the shapes of the sensor spectral responses: For camera I the responses are wider than for camera II, the discretization can thus be coarser whereas the use of camera II requires a finer sampling. Moreover, it is possible to see that $\epsilon_{\mathrm{U}}^{c}$ follows the same tendency as $\epsilon_{\mathrm{B}}^{c}$ : The adequacy becomes better as the number of wavelengths increases and stabilizes around $N=25$. For both cameras, it is thus still possible to find a limited number of wavelengths $N$ for which the errors on the simulation of the scene contribution $\mathcal{E}_{\mathrm{U}, N}^{c}$ are smaller than a few percent.

\section{Image simulation from RGB data}

The previous study has shown that it is not necessary to take into account $N=100$ wavelengths to simulate realistic underwater images, provided at least $N=25$ wavelengths are used. Nevertheless, having access to this limited number of wavelengths may still be difficult. Indeed one has usually only access to a simple RGB image of the scene, taken for example in absence of water. Only the reflectivities for the 3 spectral channels $\mathcal{R}_{R}, \mathcal{R}_{G}$ and $\mathcal{R}_{B}$ are then available to simulate what should have been observed in an underwater environment. In this case, the simplest way to simulate RGB images is to calculate only the three spectral components of the image at three wavelengths $\lambda_{R}, \lambda_{G}$ and $\lambda_{B}$. As shown in the previous section, it will necessarily decrease the accuracy of the RGB color rendering. Nevertheless, another solution can be considered. It consists in performing a first step of reconstruction in order to recover as best as possible the spectral reflectivity $\mathcal{R}_{\lambda}^{N}$ of the scene from the initial RGB data $\left\{\mathcal{R}_{R}, \mathcal{R}_{G}, \mathcal{R}_{B}\right\}$. In this section, these two approaches are detailed. They are 
respectively referred as the simple RGB and reconstruction approaches and their accuracies are compared.

\section{A. The simple $R G B$ and reconstruction approaches}

On the one hand, with the simple RGB approach, the three reflectivities $\left\{\mathcal{R}_{R}, \mathcal{R}_{G}, \mathcal{R}_{B}\right\}$ are directly used to simulate the underwater color image. In this case, following Eq. (8), the underwater image obtained in channel $c \in\{R, G, B\}$ is denoted $\mathcal{E}_{\text {rgb }}^{c}$ and reads

$$
\mathcal{E}_{\mathrm{rgb}}^{c}(x, y)=\mathcal{E}_{\mathrm{u}, \lambda_{c}}(x, y)+\mathcal{E}_{\mathrm{b}, \lambda_{c}}(x, y)
$$

where $\lambda_{c} \in\left\{\lambda_{R}, \lambda_{G}, \lambda_{B}\right\}$. Similarly the corresponding backscattering contribution is equal to $\mathcal{E}_{\mathrm{U}, \mathrm{rgb}}^{c}(x, y)=\mathcal{E}_{\mathrm{u}, \lambda_{c}}(x, y)$ and the scene contribution to $\mathcal{E}_{\mathrm{B}, \mathrm{rgb}}^{c}(x, y)=\mathcal{E}_{\mathrm{b}, \lambda_{c}}(x, y)$.

On the other hand, the reconstruction approach assumes that the images can be simulated from a spectral reflectance map with $N$ spectral channels $\left\{\hat{\mathcal{R}}_{1}, \hat{\mathcal{R}}_{2}, \cdots, \hat{\mathcal{R}}_{N}\right\}$ that have been recovered from the RGB data $\mathcal{R}_{R}, \mathcal{R}_{G}$ and $\mathcal{R}_{B}$. Retrieving these $N$ channels consists in determining a matrix $\mathbf{H}$ so that, for a given pixel with coordinates $(x, y), \hat{\mathbf{r}}=\mathbf{H m}$ where $\hat{\mathbf{r}}=\left[\hat{\mathcal{R}}_{1}(x, y), \cdots, \hat{\mathcal{R}}_{N}(x, y)\right]^{T}$ is the reconstructed spectrum, $\mathbf{m}=\left[\mathcal{R}_{R}(x, y), \mathcal{R}_{G}(x, y), \mathcal{R}_{B}(x, y)\right]^{T}$ and ${ }^{T}$ is the transpose operator. Although various approaches can be envisaged to recover the whole reflectivity spectrum [33,34], theoretical discussion about the best way to solve this under-determined inversion problem is out of the scope of this paper. As mentioned in Ref. [33], the spectral behavior of the reflectivities $\left\{\hat{\mathcal{R}}_{1}, \hat{\mathcal{R}}_{2}, \cdots, \hat{\mathcal{R}}_{N}\right\}$ is generally smooth. Therefore the smoothing inverse approach detailed in Ref. $[33,34]$ has been chosen in the following since it is also known to provide good reconstruction results when spectral responses with large bandwidth are used [34], as in the case of camera I and II. This approach consists in choosing, among all the solutions $\hat{\mathbf{r}}$ satisfying $\mathbf{m}=\Theta \hat{\mathbf{r}}$, the solution with minimal Laplacian norm, where $\Theta=\Delta \lambda\left[\phi^{R}, \phi^{G}, \phi^{B}\right]^{T}$ is the spectral response matrix. This leads to $[33,35]$

$$
\hat{\mathbf{r}}=\mathbf{G}^{-1} \Theta^{T}\left(\Theta \mathbf{G}^{-1} \Theta^{T}\right)^{-1} \mathbf{m}
$$

with $\mathbf{G}=\left[\mathbf{D}^{T} \mathbf{D}+\epsilon \mathbf{I}\right]$ where $\mathbf{I}$ is the $N \times N$ identity matrix, $\mathbf{D}$ is the matrix so that $\mathbf{D} \hat{\mathbf{r}}$ corresponds to the second derivative of $\hat{\mathbf{r}}$,

$$
\mathbf{D}=\left[\begin{array}{cccccccc}
1 & -2 & 1 & 0 & \ldots & \ldots & \ldots & 0 \\
0 & 1 & -2 & 1 & 0 & & & \vdots \\
0 & 0 & 1 & -2 & 1 & \ddots & & \vdots \\
0 & & \ddots & \ddots & \ddots & \ddots & 0 & \vdots \\
\vdots & & & 0 & 1 & -2 & 1 & 0 \\
0 & \ldots & \ldots & \ldots & 0 & 1 & -2 & 1
\end{array}\right]
$$


and $\epsilon$ is small regularization constant $\left(\epsilon=10^{-14}\right.$ in the simulations) to prevent $\mathbf{G}$ from being singular (see details in Ref. [34]).

According to Eq. (8), the underwater image in channel $c \in\{R, G, B\}$ simulated using this reconstruction approach is thus equal to

$$
\hat{\mathcal{E}}_{N}^{c}(x, y)=\hat{\mathcal{E}}_{\mathrm{U}, N}(x, y)+\mathcal{E}_{B, N}(x, y)
$$

where $\hat{\mathcal{E}}_{\mathrm{U}, N}(x, y)$ corresponds to the scene contribution simulated from the previous estimated reflectance map $\left\{\hat{\mathcal{R}}_{n}(x, y)\right\}$. Moreover, since the backscattering contribution $\mathcal{E}_{B, N}(x, y)$ is independent of the scene, it is still calculated according to the same method as in section 2.

\section{B. Comparison of the two approaches}

In this subsection, it is proposed to study the precision of these two approaches. In order to have a reference to compare the adequacy of the two methods, the theoretical scene of subsection 1.B is once again considered and the images $\mathcal{E}_{\bullet}^{c}, N=100$ of section 2 are still taken as the ground truth $\mathcal{E}_{\bullet}^{c}$,true. However it is now assumed that only the RGB maps shown in Fig. 4 are available for underwater image simulation, i.e. one has only access to $\left\{\mathcal{R}_{R}, \mathcal{R}_{G}, \mathcal{R}_{B}\right\}$ with

$$
\mathcal{R}_{c}(x, y)=\sum_{n=1}^{N} \phi^{c}\left(\lambda_{n}\right) \mathcal{R}_{\lambda_{n}}(x, y)
$$

with $c \in\{R, G, B\}$. Moreover, for the simple RGB approach, $\lambda_{\mathrm{R}}=619 \mathrm{~nm}, \lambda_{\mathrm{G}}=534 \mathrm{~nm}$, $\lambda_{\mathrm{B}}=459 \mathrm{~nm}$ are chosen, which corresponds to the wavelengths of the camera response maxima.

Fig. 9 shows examples of simulation using either the simple RGB approach or the reconstruction approach for both types of water and both cameras. All simulations were performed for $Z_{o}=7 \mathrm{~m}$. As it can be seen, the two approaches give different visual rendering, especially for camera I. For a better analysis of these results, the irradiance in the red and blue channels for the 20 patches have been plotted in Fig. 10 for camera I. These profiles have been obtained using either the ground truth shown in Fig. 5 (plain curves), the simple RGB approach (dots with dashed curves) or the reconstruction approach with $N=100$ (crosses), in the case of three types of medium (air, type 1 water and type 2 water).

As it can be seen, the three approaches are equivalent in the absence of water (cf. Fig. 10 row 1), but the simple RGB strategy may lead to image spectral components that strongly differ from the ground truth in the presence of water. Indeed, in the red channel (Fig. 10.(a)), the simple RGB approach tends to underestimate the contrast of the patches in type 1 water (cf. Fig. 10.(a) row 2). In this case, the simple RGB strategy predicts a RGB color image in which most of the details may be lost in the red channel in the presence of noise (i.e. the red channel is assumed to contain no more information on the scene) whereas the different patches 
can still be distinguished on the ground truth red channel. Similarly in water of type 2, Fig. 10 (row 3) shows that the RGB approach miscalculates the backscattering contribution either by underestimating it in the red channel (Fig. 10.(a) row 3) or by overestimating it in the blue channel (Fig. 10.(b) row 3). These phenomena are due to the shape of the spectral responses of camera I: indeed the secondary maxima of $\phi_{\lambda}^{R}, \phi_{\lambda}^{G}$ and $\phi_{\lambda}^{B}$ can not be taken into account by the simple RGB approach. This remark highlights the fact that modeling the spectral response of the sensor is necessary to have an adequate color rendering. On the contrary, even if the reconstruction of the spectral reflectivities $\hat{\mathcal{R}}_{\lambda}$ from $\left\{\mathcal{R}_{R}, \mathcal{R}_{G}, \mathcal{R}_{B}\right\}$ cannot be very accurate, the RGB image simulated with the reconstruction approach is close to the ground truth (see crosses in Fig. 10 curves compared to ground truth plain lines).

These conclusions are confirmed by the calculation of the errors $\epsilon_{\mathrm{U}}^{c}$ and $\epsilon_{\mathrm{B}}^{c}$ performed when using the simple RGB approach (cf. Fig. 6, Fig. 7 and Fig. 8, black and gray triangles for errors in the red channel). As it can be seen the errors are higher with this approach compared to the case where images are simulated with $N \geq 25$. This is true for simulations using both cameras as errors can be almost one order of magnitude higher compared to simulations using the direct multispectral approach (detailed in section 2 and which assumes that the spectral reflectance of the scene is perfectly known). On the other hand, when image simulations are performed with the reconstruction approach (black and gray dots in Fig. 7 and Fig. 8), errors have a behavior very similar to the case where the scene reflectance is assumed to be perfectly known and decrease as the number of reconstructed wavelengths increases. Of course, contrary to the case of a perfectly known reflectance, errors do not converge toward zero since the reconstruction method cannot retrieve exactly the initial spectrum, but the errors still stabilize for $N \geq 25$ and remain lower than errors due to the simple RGB approach. Thus, even if only RGB data of the scene are available, retrieving the spectral information of the scene seems to be generally beneficial for RGB underwater image formation compared to the simpler RGB approach.

\section{C. Example on real data}

All the experiments presented in the study so far are based on a scene with perfectly known characteristics. Fig 11.(a) now illustrates an "homemade scene" of unknown spectral reflectance taken with a standard camera. Assuming a type II camera, underwater images have been simulated for type 1 water and $Z_{o}=7 \mathrm{~m}$ with the same experimental conditions as in the previous sections. Fig. 11.(c) and Fig. 11.(b) respectively show the results obtained with the simple RGB approach and the reconstruction approach with $N=25$ (i.e. 25 spectral reflectance values have been reconstructed from the initial RGB channels of the scene). In Fig. 11.(d)-(f) the red channels of the corresponding images are displayed.

Although both simulation methods give quite similar visual color rendering, more critical 
differences can be seen on the red channels. For example, with the simple RGB approach (Fig. 11.(f)), the red component of non purely red objects seems to be more attenuated compared to the image obtained with the reconstruction approach (Fig. 11.(e)). In particular, this leads to a darker notebook cover and a weaker contrast between the red pen and the background on the left of the image (see red frames). Indeed, in the case of the reconstruction approach, all the wavelengths integrated in the red channel are not as attenuated as the single red wavelength used in the simple RGB approach, leading to higher red intensity in Fig. 11.(e). Therefore, assuming that one wants to restore the image colors for example, a given technique may lead to different restorations depending on the simulation approach used to generate the images.

Moreover comparing the red channels of the initial RGB image and of the simulated image obtained with the simple RGB approach (respectively Fig. 11.(d) and Fig. 11.(f)), one can see that both images are very close. In the case of the simple RGB approach, light propagation in clear water (for $Z_{o}=7 \mathrm{~m}$ ) results mainly in a simple attenuation of the red component. Thus, in the case of color restoration, a simple algorithm, that could be considered to restore Fig. 11.(f), consists in estimating a multiplicative coefficient for each channel in order to compensate for the attenuation. However, for the image obtained with the reconstruction approach which has been shown to lead to more realistic color rendering (cf. subsection 3.B), such a simple method cannot be sufficient since this simulated image displays a more complex spectral behavior (cf. Fig. 11.(e)). Indeed, contrary to Fig. 11.(f) where the pen and notebook cover have different intensities (as in Fig. 11.(d)), both objects have similar gray levels in Fig. 11.(e); this behavior cannot be summed up to a simple attenuation factor.

All these observations highlight a key issue: Since a given situation may lead to different diagnoses depending on the way the image is simulated, it may completely bias the characterization of image processing algorithms.

\section{Conclusion}

In conclusion, we have presented an underwater color image simulator which extends standard models to RGB color image simulation, taking into account the spectral characteristics of all the set-up parameters. It particularly illustrates that choices about detector spectral responses can clearly impact the visual aspect of the images and may not be neglected for a correct color rendering. Moreover it has been shown that, depending on the way the images are simulated, the behaviors of the RGB channels can greatly differ. This is a key point since it may strongly impact the diagnosis that can be made on the analysis of the main source of image degradation, especially in the perspective of color restoration.

Furthermore the analysis shows that a complete knowledge of the spectral information of the scene is advantageous but not mandatory. In the examples discussed in the paper, it was 
possible to find a limited number of wavelengths (typically $N \approx 25$ ) to use in the simulations so that the errors on the simulated images were small enough compared to the ground truth model.

We have also shown that even if only RGB data are available to simulate an underwater measurement, a preprocessing step allowing one to recover more spectral information about the scene intrinsic reflectance is generally beneficial for the simulation.

One of the main perspectives of this work is to use a simulator to characterize different color restoration algorithms. Moreover all the simulations presented in this study use the same VSF model which relies on the data from Ref. [2]. These data are still used as reference in literature [27-30] but are known to have limitations [31,32]. Another perspective is thus to study the impact of the various VSF models and measurements on the color degradation of RGB underwater. Finally the reconstruction technique presented in the analysis uses a standard smoothing inverse approach, but other methods could be used such as Wiener filtering [33]. Studying the impact of the reconstruction method on the simulation precision thus appears also as an interesting perspective.

\section{Acknowledgments}

The authors would like to thank Philippe Réfrégier from Institut Fresnel for fruitful discussions and contributions, as well as Malik Chami and Kerstin Ebert from Laboratoire d'Océanographie de Villefranche-sur-Mer for advice concerning water optical properties. This

work has been partially supported by French National Research Agency (ANR) through COSINUS program (project OSIFIOST n ${ }^{\circ}$ ANR-08-COSI-008), with an additional support through the Pôle Mer PACA.

\section{References}

1. S. Duntley, "Underwater lighting by submerged lasers and incandescent sources," Tech. rep., Scripps. Inst. of Oceanography, San Diego (1971).

2. T. Petzold, "Volume scattering functions for selected ocean waters," Tech. rep., Scripps. Inst. of Oceanography, San Diego (1972).

3. W. Wells, "Theory of small angle scattering," AGARD conference proceedings 81, 1-20 (1973).

4. B. McGlamery, "Computer analysis and simulation of underwater camera system performance," Tech. rep., Scripps. Inst. of Oceanography, San Diego (1975).

5. J. Jaffe, J. Mclean, M. Strand, and K. Moore, "Underwater Optical Imaging: Status and Prospects," Oceanography 14, 64-75 (2001). 
6. D. Kocak, F. Dalgleish, and F. Caimi, "A focus on recent developments and trends in underwater imaging," Marine Technology 42, 52-67 (2008).

7. R. Schettini and S. Corchs, "Underwater Image Processing: State of the Art of Restoration and Image Enhancement Methods," EURASIP Journal on Advances in Signal Processing 2010 (2010).

8. L. Mertens and F. Replogle, Jr., "Use of point spread and beam spread functions for analysis of imaging systems in water," Journal of the Optical Society of America 67, 1105-1117 (1977).

9. B. McGlamery, "A computer model for underwater camera systems," in "Proceedings of the SPIE, Vol. 0208," (1979), pp. 221-231.

10. J. Jaffe, "Computer Modeling and the Design of Optimal Underwater Imaging Systems," IEEE Journal of Oceanic Engineering 15, 101-111 (1990).

11. S. Bazeille, I. Quidu, L. Jaulin, and J.-P. Malkasse, "Automatic underwater image preprocessing," in "CMM06," (Brest, 2006), p. 8.

12. E. Trucco and A. Olmos-Antillon, "Self-Tuning Underwater Image Restoration," IEEE Journal of Oceanic Engineering 31, 511-519 (2006).

13. T. Treibitz and Y. Schechner, "Active polarization descattering." IEEE transactions on pattern analysis and machine intelligence 31, 385-99 (2009).

14. Y. Schechner and N. Karpel, "Recovery of Underwater Visibility and Structure by Polarization Analysis," IEEE Journal of Oceanic Engineering 30, 570-587 (2005).

15. J. Ahlén, E. Bengtsson, and D. Sundgren, "Evaluation of underwater spectral data for colour correction applications," in "Proceedings of the 5th WSEAS International Conference on Circuits, Systems, Electronics, Control \& Signal Processing," (World Scientific and Engineering Academy and Society (WSEAS), Dallas, 2006), pp. 321-326.

16. M. Chambah, D. Semani, A. Renouf, P. Courtellemont, and A. Rizzi, "Underwater color constancy: Enhancement of automatic live fish recognition," Color Imaging IX: Processing, Hardcopy, and Applications 5293, 157-168 (2003).

17. K. Iqbal, R. Abdul Salam, M. Osman, and A. Talib, "Underwater Image Enhancement Using An Integrated Colour Model." IAENG International Journal of Computer Science 34, 239-244 (2007).

18. F. Petit, A.-S. Capelle-Laize, and P. Carre, "Underwater image enhancement by attenuation inversion with quaternions," IEEE International Conference on Acoustics Speech and Signal Processing (2009) pp. 1177-1180 (2009).

19. L. Torres-Méndez and G. Dudek, "Color correction of underwater images for aquatic robot inspection," in "Energy Minimization Methods in Computer Vision and Pattern Recognition," (Springer, 2005), pp. 60-73.

20. W. Hou, D. Gray, A. Weidemann, and R. Arnone, "Comparison and validation of point 
spread models for imaging in natural waters." Optics Express 16, 9958-65 (2008).

21. S. Negahdaripour, H. Zhang, and X. Han, "Investigation of photometric stereo method for 3-d shape recovery from underwater imagery," in "MTS/IEEE Oceans," (Marine Technol. Soc, Biloxi, 2002), pp. 1010-1017.

22. A. Pentland, "A new sense for depth of field," IEEE transactions on pattern analysis and machine intelligence 9, 523-531 (1987).

23. G.C. Holst, and T.S. Lomheim, CMOS/CCD Sensors and Camera Systems (SPIE Press, JCD Publishing, Bellingham, 2007).

24. J. Ahlén, D. Sundgren, and E. Bengtsson, "Application of underwater hyperspectral data for color correction purposes," Pattern Recognition and Image Analysis 17, 170173 (2007).

25. E. Valero, J. Nieves, S. Nascimento, K. Amano, and D. Foster, "Recovering spectral data from natural scenes with an RGB digital camera and colored filters," Color Research \& Application 32, 352-360 (2007).

26. R. Clark, G. Swayze, R. Wise, E. Livo, T. Hoefen, R. Kokaly, and S. Sutley, "USGS digital spectral library splib06a, U.S. Geological Survey, Digital Data Series 231, http://speclab.cr.usgs.gov/spectral.lib06," (2007).

27. C.D. Mobley, B. Gentili, H.R. Gordon, Z. Jin, G.W. Kattawar, P. Reinersman, K. Stamnes, and R.H.Stavn, "Comparison of numerical models for computing underwater light fields",Applied Optics 32, 7484-7504 (1993).

28. H. Loisel and D. Stramski, "Estimation of the inherent optical properties of natural waters from the irradiance attenuation coefficient and reflectance in the presence of Raman scattering", Applied Optics 39, 3001-3011 (2000).

29. J. Chowdhary, B. Cairns and L.D. Travis, "Case Studies of Aerosol Retrievals over the Ocean from Multiangle, Multispectral Photopolarimetric Remote Sensing Data" 59, 383-397 (2002).

30. C. Mobley, Light and Water (Academic Press, London, 1994), united kin ed.

31. M.E. Lee and R.L. Lewis, A New Method for the Measurement of the Optical Volume Scattering Function in the Upper Ocean, Journal of Atmospheric and oceanic technology 20, 563-571 (2003).

32. Y.C. Agrawal, The optical volume scattering function: Temporal and vertical variability in the water column off the New Jersey coast, Liminology and Oceanography 50, 17871794 (2005).

33. W. Pratt and C. Mancill, "Spectral estimation techniques for the spectral calibration of a color image scanner." Applied optics 15, 73-5 (1976).

34. L.W. MacDonald and M.R. Luo, Colour Imaging: Vision and Technology (Wiley, Chichester, 1999). 
35. C.R. Rao and S.K. Mitre, Generalized Inverse of a matrix and its application, Proceedings of the 6th Berkeley Symposium of Mathematical Statistics and Probability, Vol. I, 601-620 (1972).
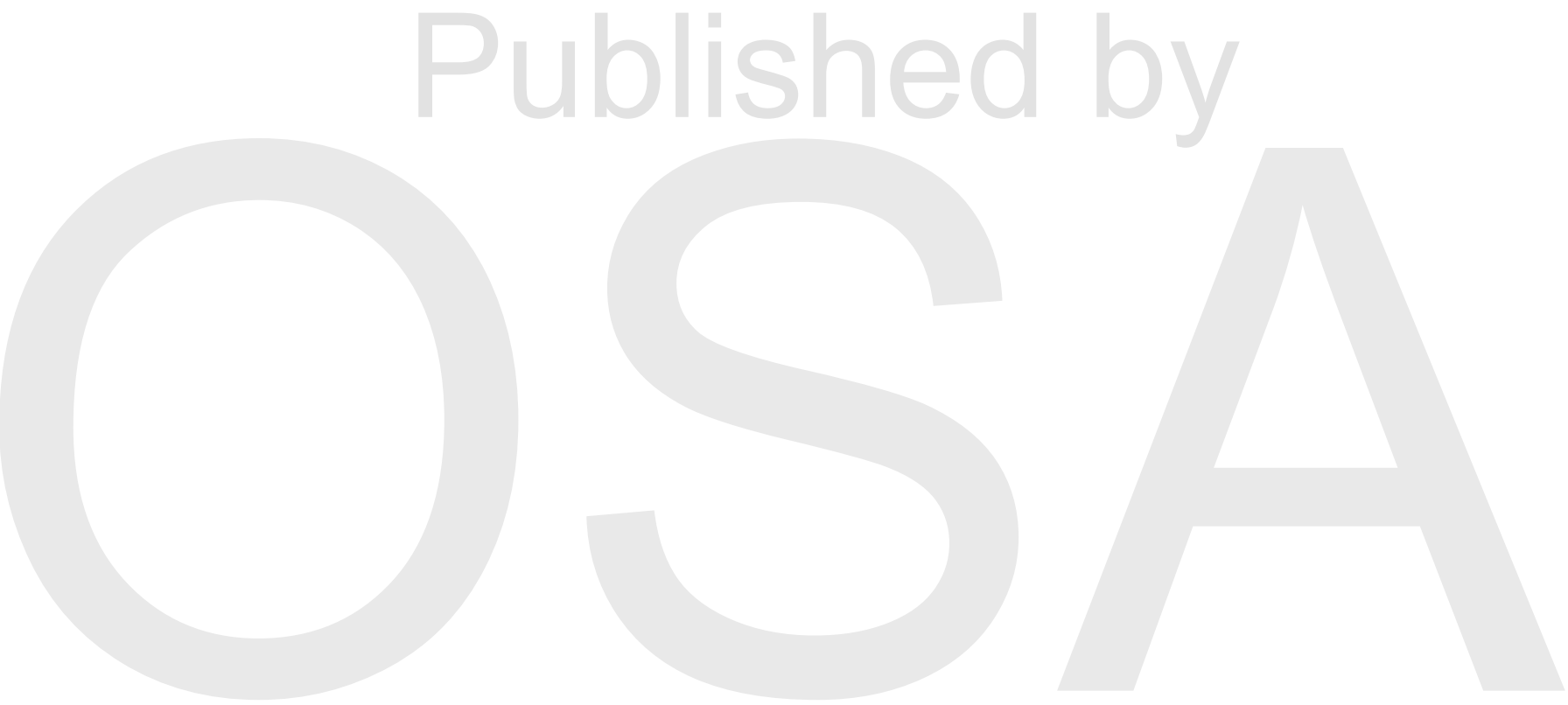


\section{List of Figure Captions}

Fig. 1. Geometry and coordinate system of the model used for this study. The scene is assumed to be parallel to the detector plane. (a) For general calculation. (b) For the backscattering contribution calculation.

Fig. 2. (a) Inherent optical properties for type 1 water (clear water) used in the study: Beam absorption coefficient $a_{\lambda}$, scattering coefficient $b_{\lambda}$ and total attenuation coefficient $c_{\lambda}$. (b) Idem for type 2 water (turbid water). (c) Values of $p(\varphi)$ used to calculated the volume scattering function (data taken from Ref. [4]).

Fig. 3. [color on-line] Spectral responses of the cameras used for the study. They were adapted (a) from Ref. [24] for camera I and (b) from Ref. [25] for camera II.

Fig. 4. [color on-line] RGB representation of the reference scene with a known spectral reflectance. The reflection coefficient for each patch is perfectly known and taken from Ref. [26]. The scene was imaged in air with camera I (a) and camera II (b). Image size is 46x37 pixels. Fig. 5. [color on-line] Visual aspect of scene of Fig.4 in subsea environments for $Z_{o}=7 \mathrm{~m}$. Upper row: The scene was imaged using camera I. Bottom row: Idem with camera II. Left column: Results of simulation for type 1 water. Right column: Idem for type 2 water.

Fig. 6. Error $\epsilon_{\mathrm{B}}^{c}$ on the simulation of the backscattering contribution in type 1 water as a function of the number $N$ of wavelengths for $Z_{o}=15 \mathrm{~m}$. The results are plotted as a percentile ratio of $\max _{x, y} \mathcal{E}_{\mathrm{B}, \text { true }}^{c}(x, y)$ for (a) the red channel, (b) the green channel and (c) the blue channel.

Fig. 7. Error $\epsilon_{\mathrm{U}}^{R}$ on the simulation of the scene contribution in the red channel for type 1 water as a function of the number $N$ of wavelengths. The result is plotted as a percentile ratio of $\max _{x, y} \mathcal{E}_{\mathrm{U}, \text { true }}^{R}(x, y)$ and for $Z_{o}=15 \mathrm{~m}$.

Fig. 8. Same as Fig. 7 , but for type 2 water with $Z_{o}=7 \mathrm{~m}$.

Fig. 9. [color on-line] Visual aspect of scene of Fig.4 in subsea environments for $Z_{o}=7 \mathrm{~m}$. Upper row: The scene was imaged using camera I. Bottom row: Idem with camera II. Left columns: Results of simulation with for type 1 water. Right columns: Idem for type 2 water. (a), (c), (e), (g): Simulations were performed with the simple RGB approach. (b), (d), (f), (h): Idem with the reconstruction approach.

Fig. 10. Irradiance profiles of the 20 patches corresponding to the images of Fig. 4.(a) simulated for $Z_{o}=7 \mathrm{~m}$ with camera I. Row 1: Irradiance profiles in air. Row 2: Irradiance profiles in type 1 water. Row 3: Irradiance profiles in type 2 water. Plain curves: Ground truth. Dots and dashed line: Simple RGB approach. Crosses: Reconstruction approach. (a) Red channel. (b) Blue channel.

Fig. 11. [color on-line] (a) Initial RGB image (256x160 pixels) taken with a standard camera. (b) Simulated RGB image for type 1 water and a distance $Z_{o}=7 \mathrm{~m}$ with a reconstruction step and the multispectral approach $(N=25)$. (c) Idem using the simple RGB approach. 
(d), (e), (f): Red channels correspond respectively to (a), (b) and (c).
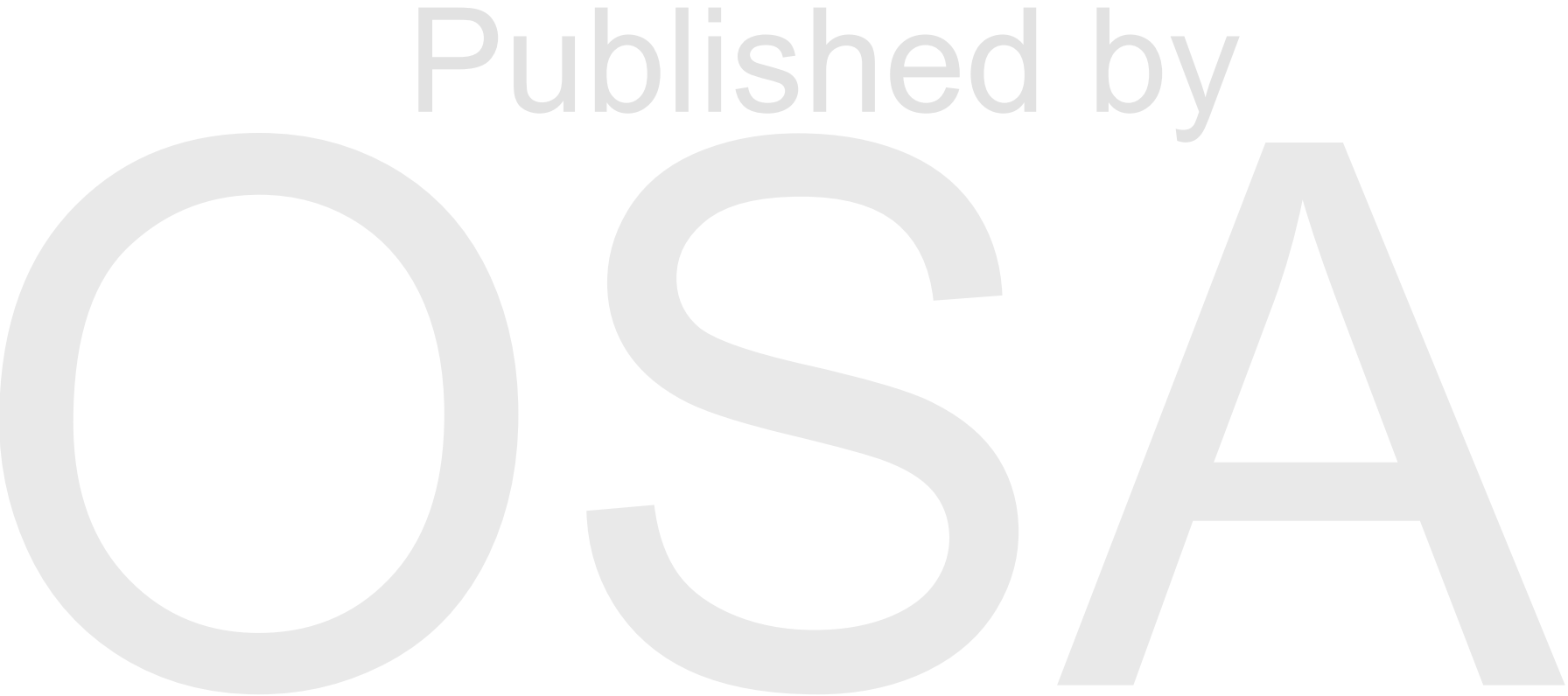


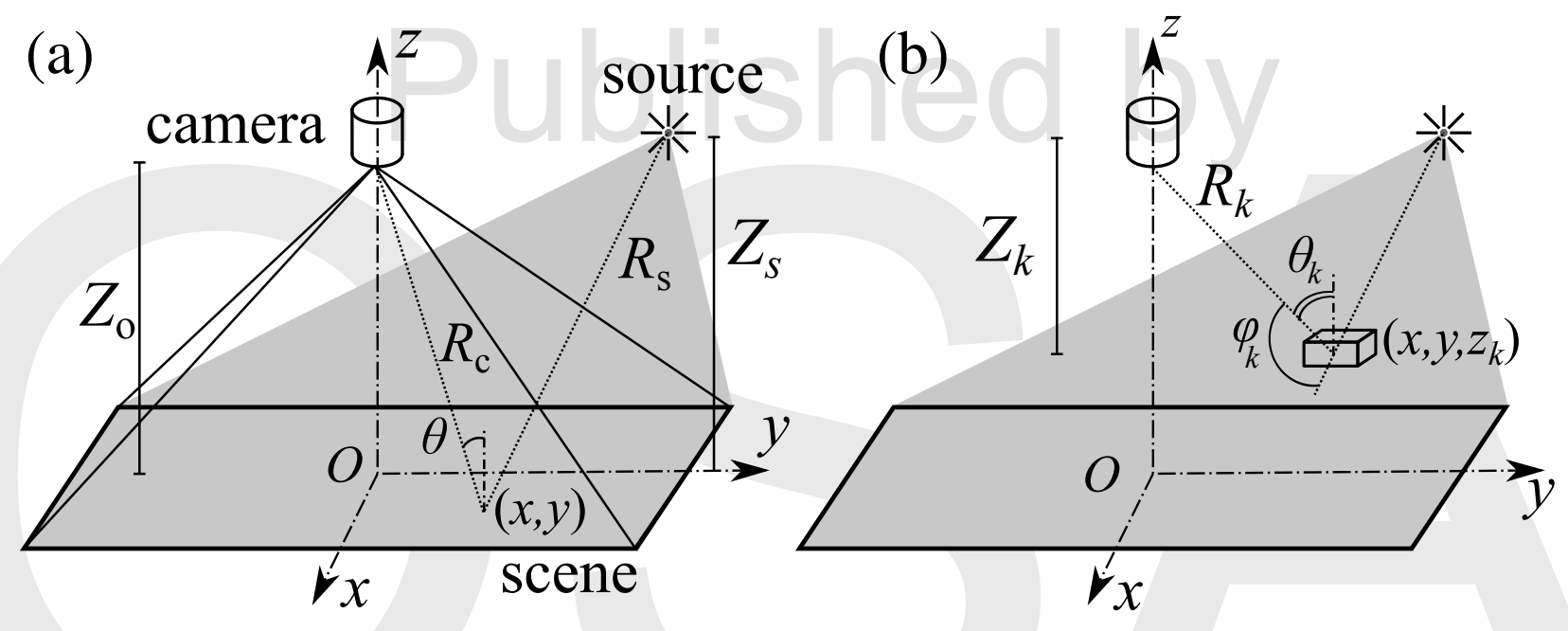

Fig. 1. Geometry and coordinate system of the model used for this study. The scene is assumed to be parallel to the detector plane. (a) For general calculation. (b) For the backscattering contribution calculation. 
(a) TYPE 1 WATER

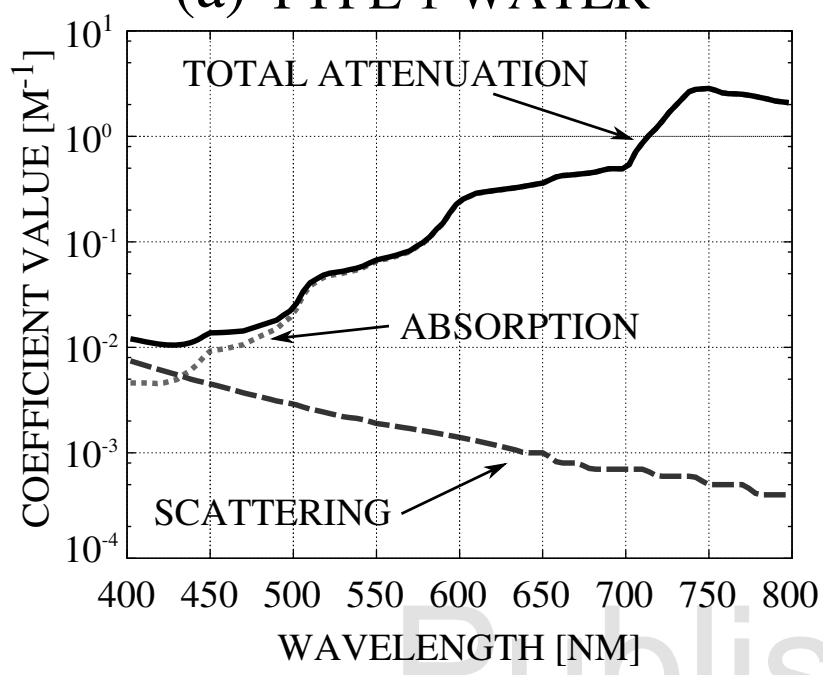

(b) TYPE 2 WATER

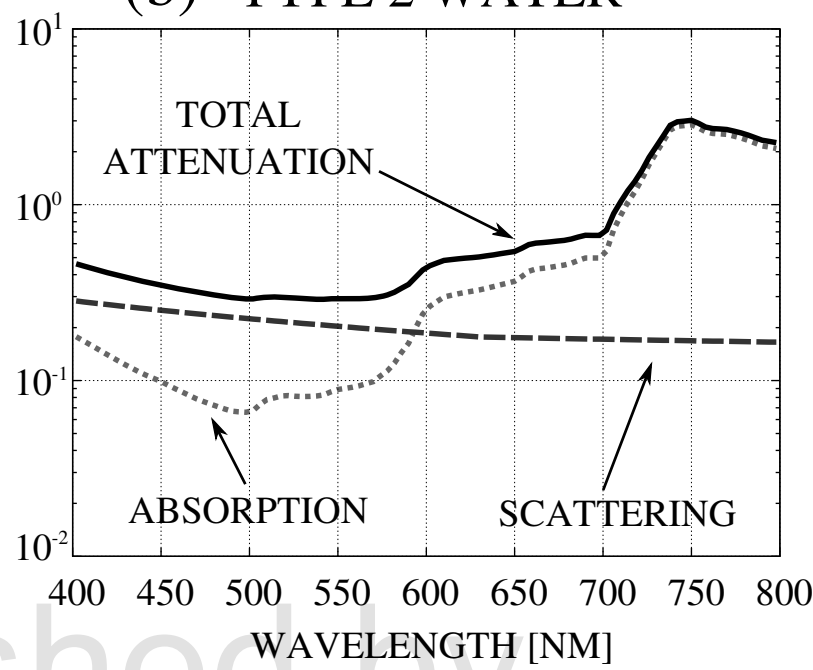

\section{(c) DATA FOR VSF CALCULATION}

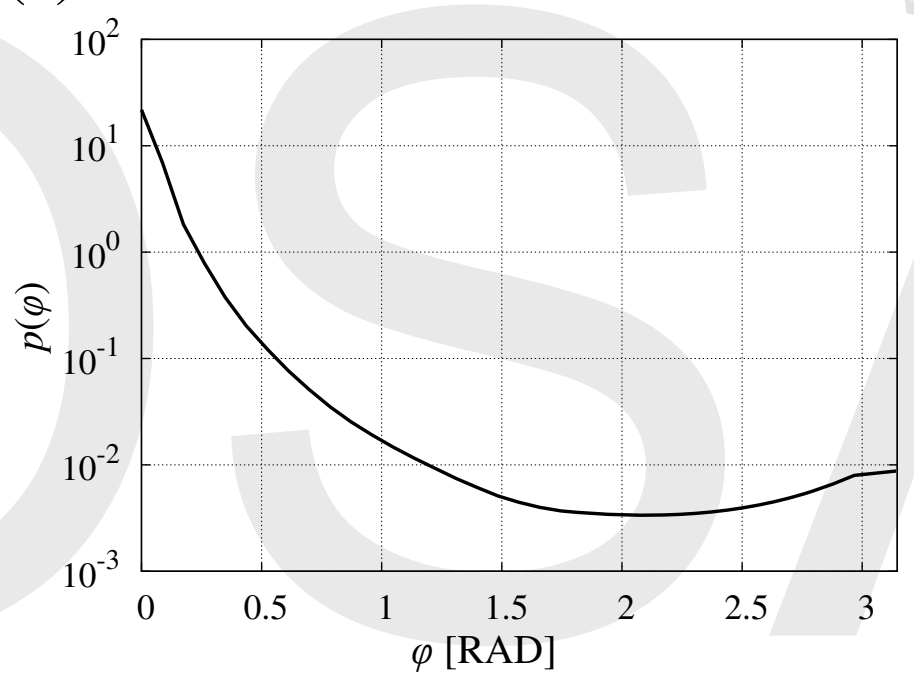

Fig. 2. (a) Inherent optical properties for type 1 water (clear water) used in the study: Beam absorption coefficient $a_{\lambda}$, scattering coefficient $b_{\lambda}$ and total attenuation coefficient $c_{\lambda}$. (b) Idem for type 2 water (turbid water). (c) Values of $p(\varphi)$ used to calculated the volume scattering function (data taken from Ref. [4]). 
(a) CAMERA I

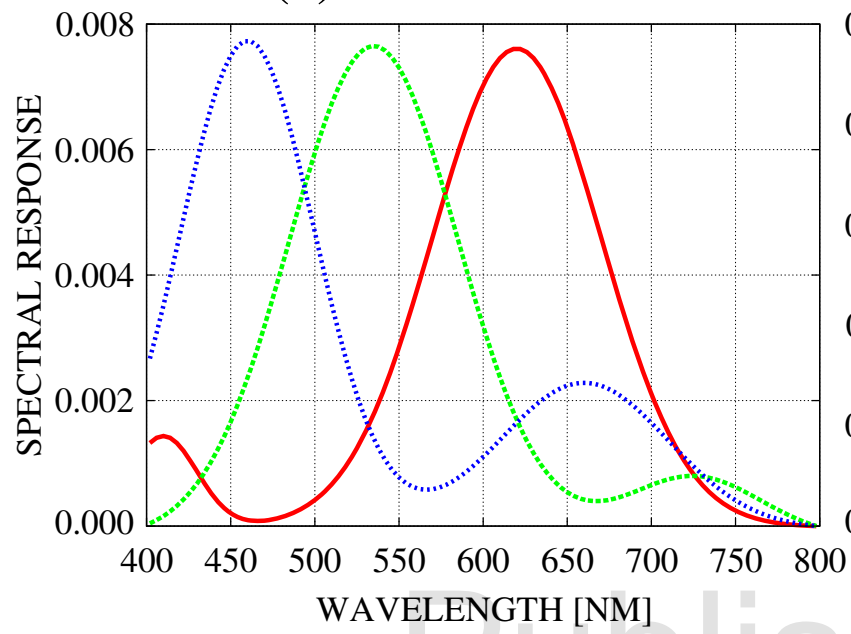

(b) CAMERA II

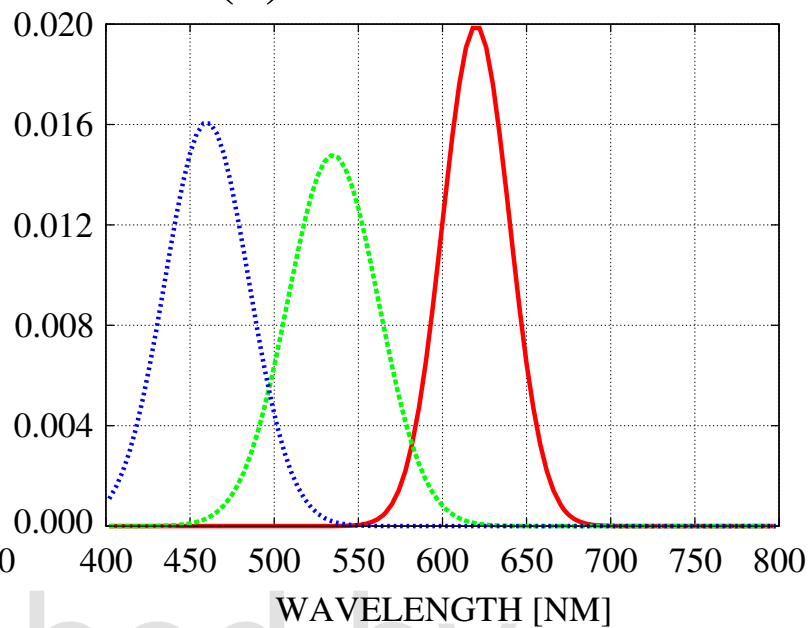

Fig. 3. [color on-line] Spectral responses of the cameras used for the study. They were adapted (a) from Ref. [24] for camera I and (b) from Ref. [25] for camera II. 


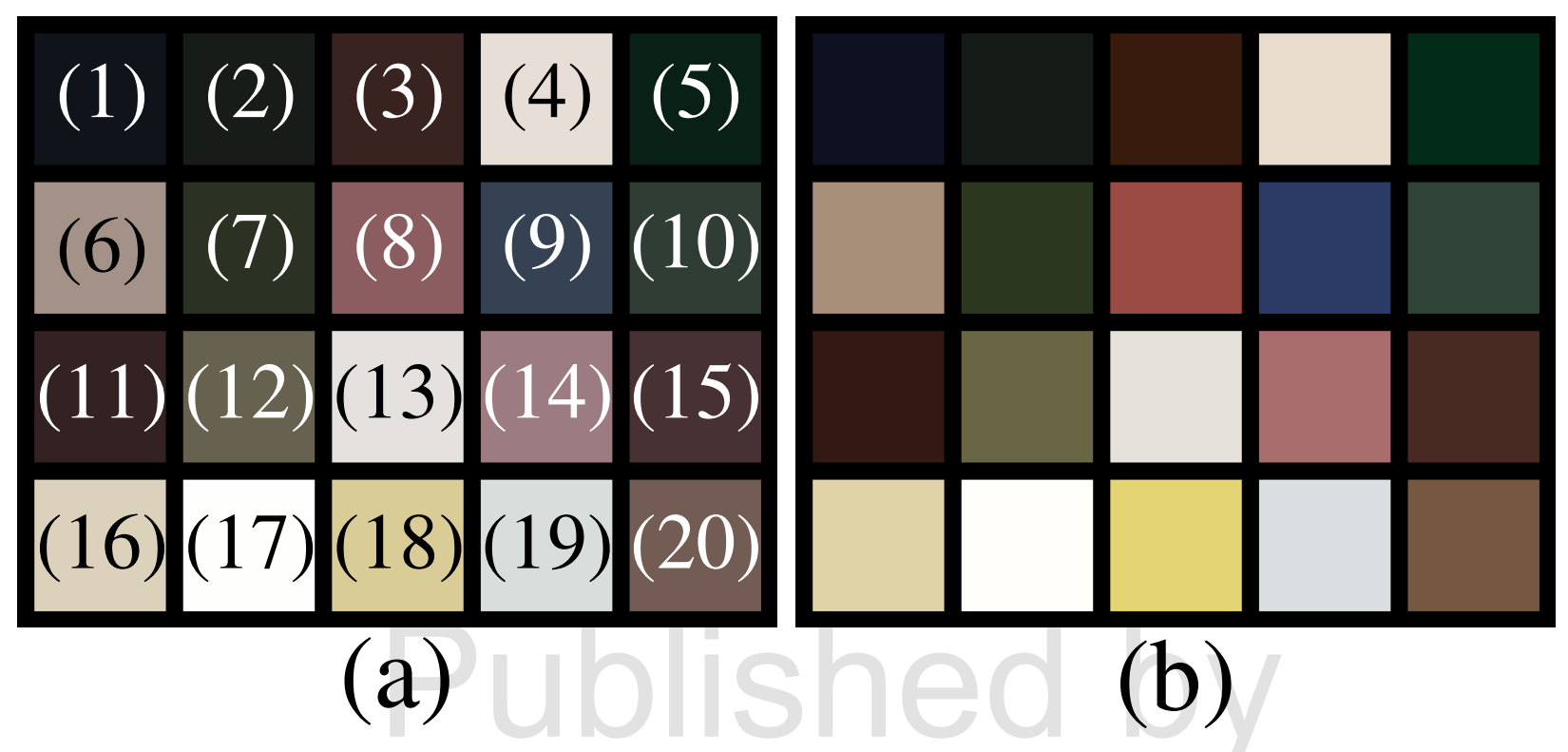

Fig. 4. [color on-line] RGB representation of the reference scene with a known spectral reflectance. The reflection coefficient for each patch is perfectly known and taken from Ref. [26]. The scene was imaged in air with camera I (a) and camera II (b). Image size is 46x37 pixels. 


\section{TYPE 1 WATER}
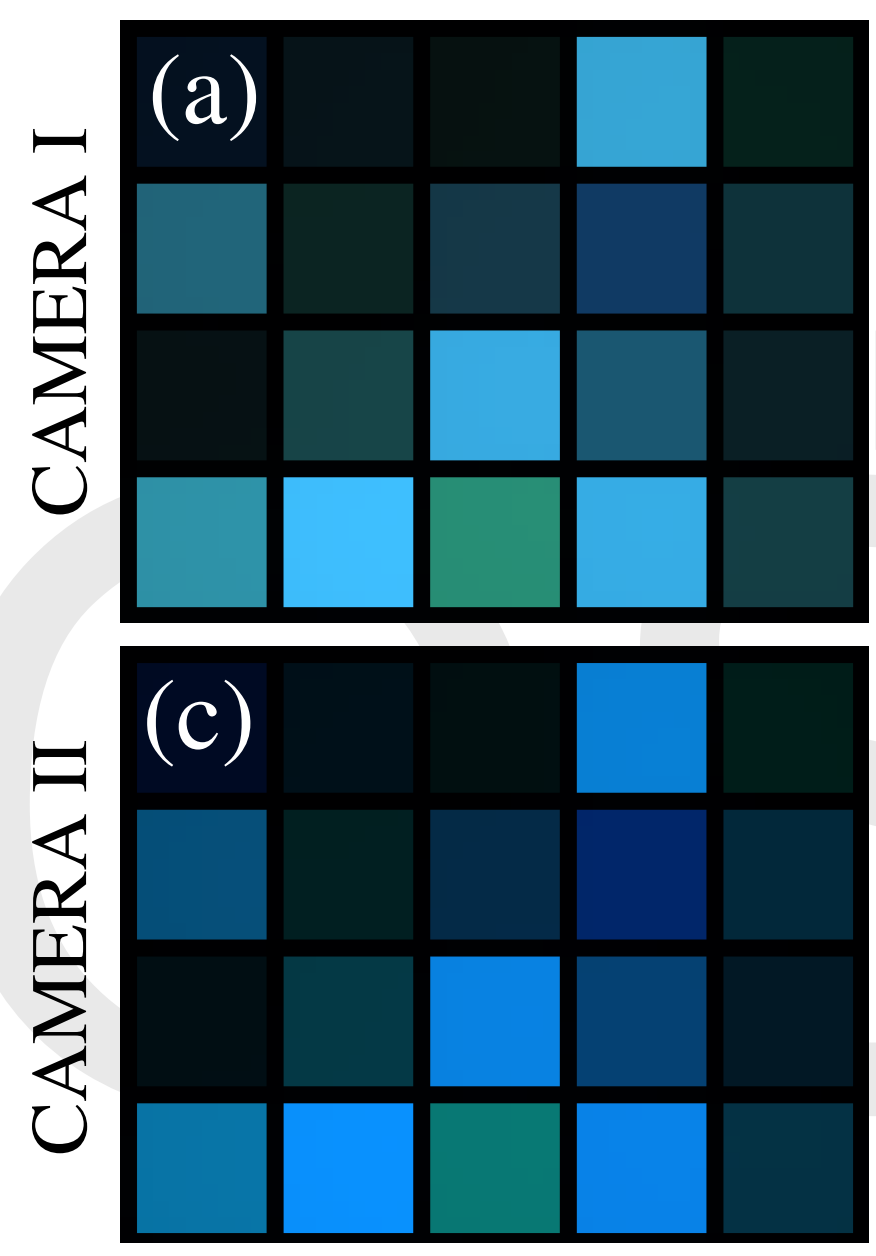

TYPE 2 WATER
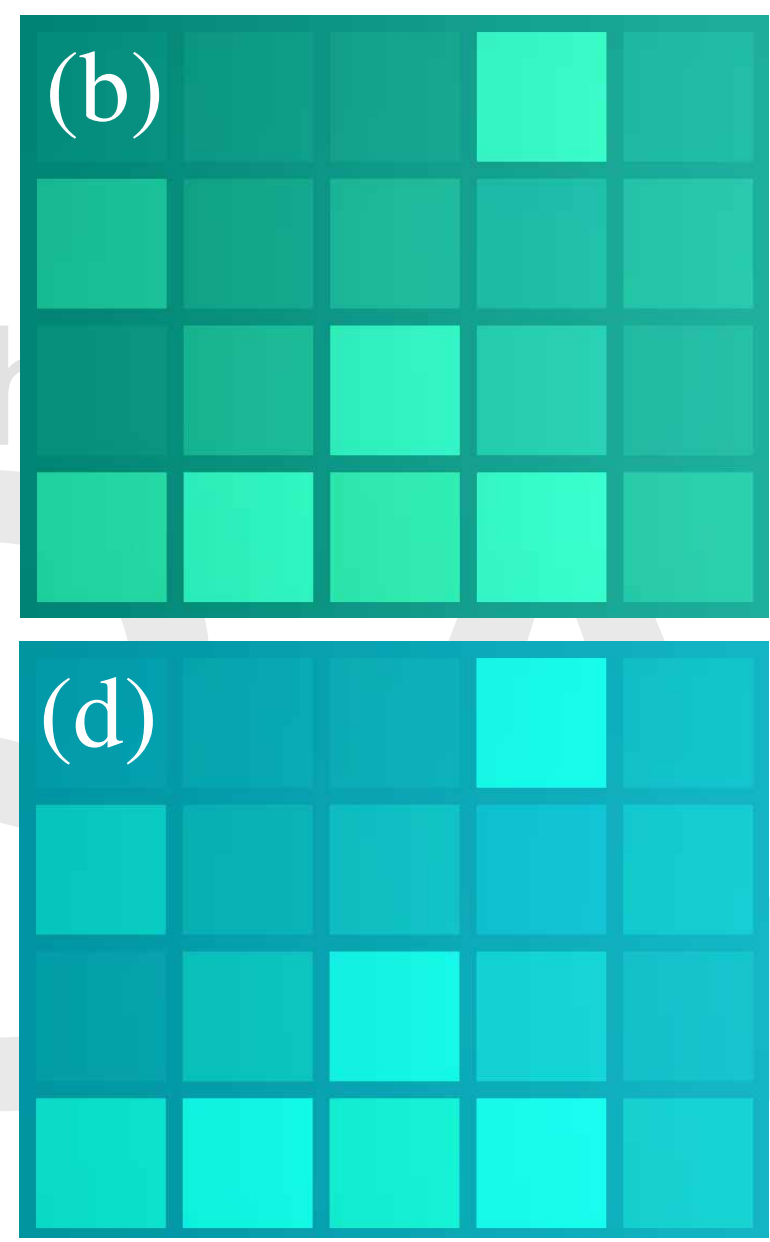

Fig. 5. [color on-line] Visual aspect of scene of Fig.4 in subsea environments for $Z_{o}=7 \mathrm{~m}$. Upper row: The scene was imaged using camera I. Bottom row: Idem with camera II. Left column: Results of simulation for type 1 water. Right column: Idem for type 2 water. 
(a) RED CHANNEL

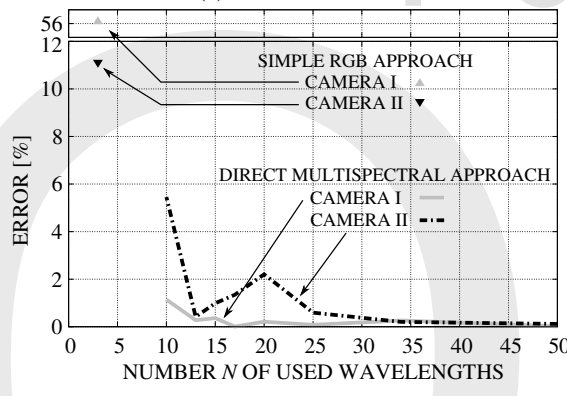

(b) GREEN CHANNEL

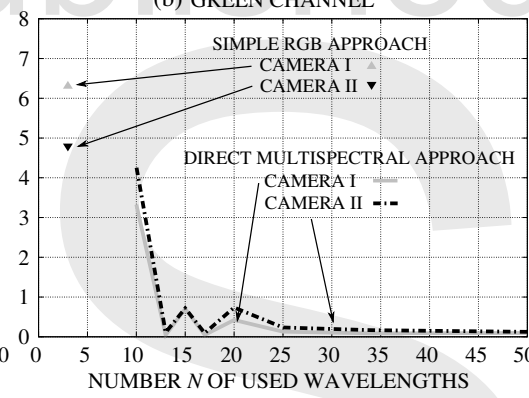

(c) BLUE CHANNEL

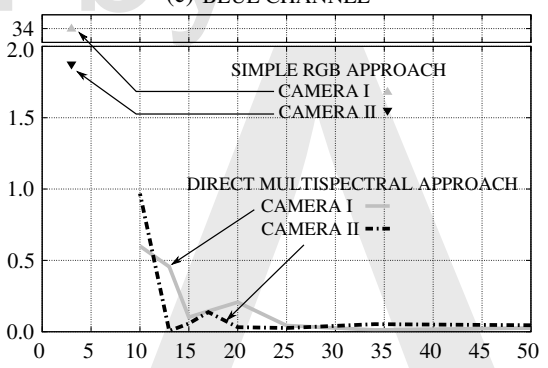

NUMBER $N$ OF USED WAVELENGTHS

Fig. 6. Error $\epsilon_{\mathrm{B}}^{c}$ on the simulation of the backscattering contribution in type 1 water as a function of the number $N$ of wavelengths for $Z_{o}=15 \mathrm{~m}$. The results are plotted as a percentile ratio of $\max _{x, y} \mathcal{E}_{\mathrm{B} \text {,true }}^{c}(x, y)$ for (a) the red channel, (b) the green channel and (c) the blue channel. 


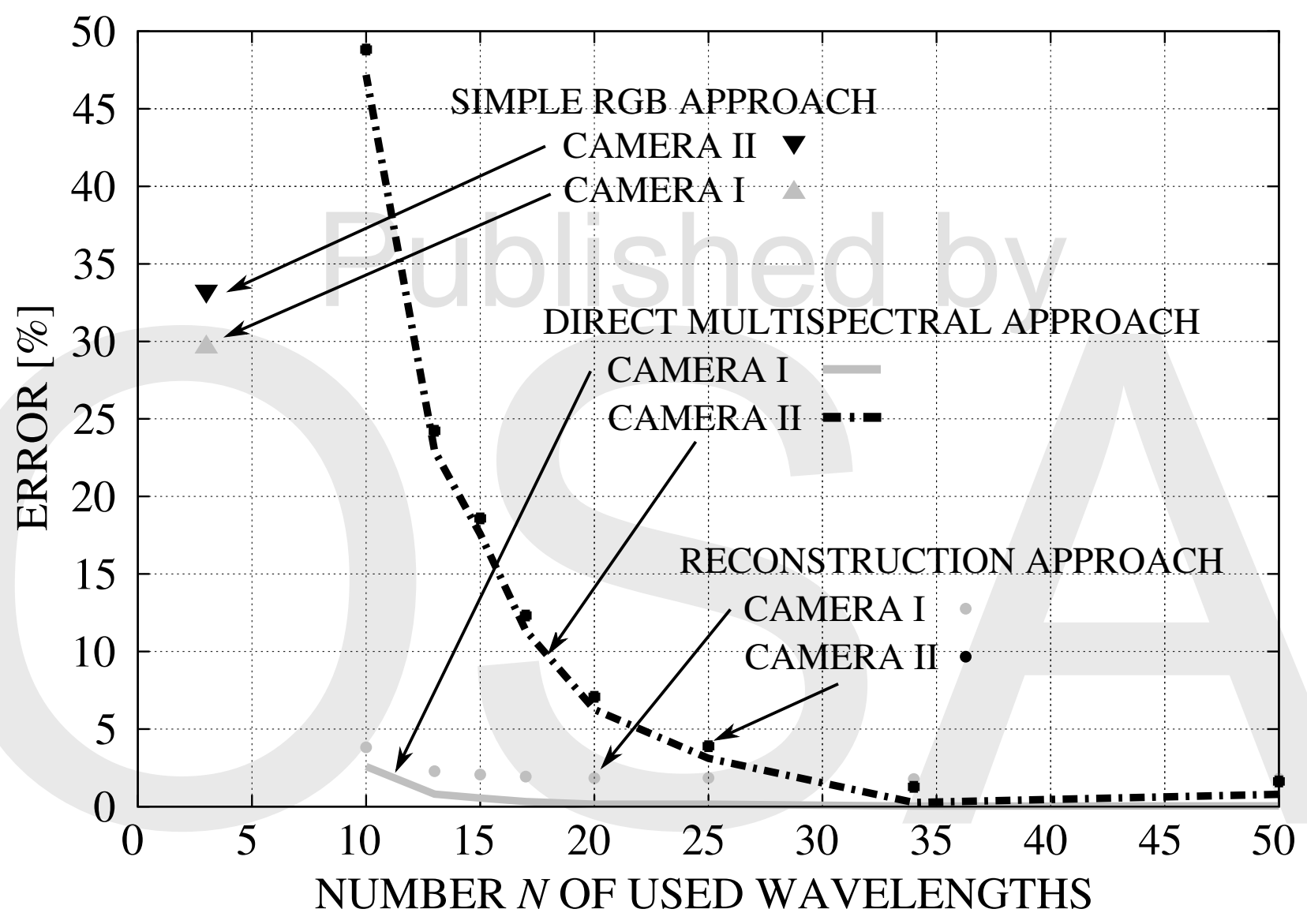

Fig. 7. Error $\epsilon_{\mathrm{U}}^{R}$ on the simulation of the scene contribution in the red channel for type 1 water as a function of the number $N$ of wavelengths. The result is plotted as a percentile ratio of $\max _{x, y} \mathcal{E}_{\mathrm{U}, \text { true }}^{R}(x, y)$ and for $Z_{o}=15 \mathrm{~m}$. 


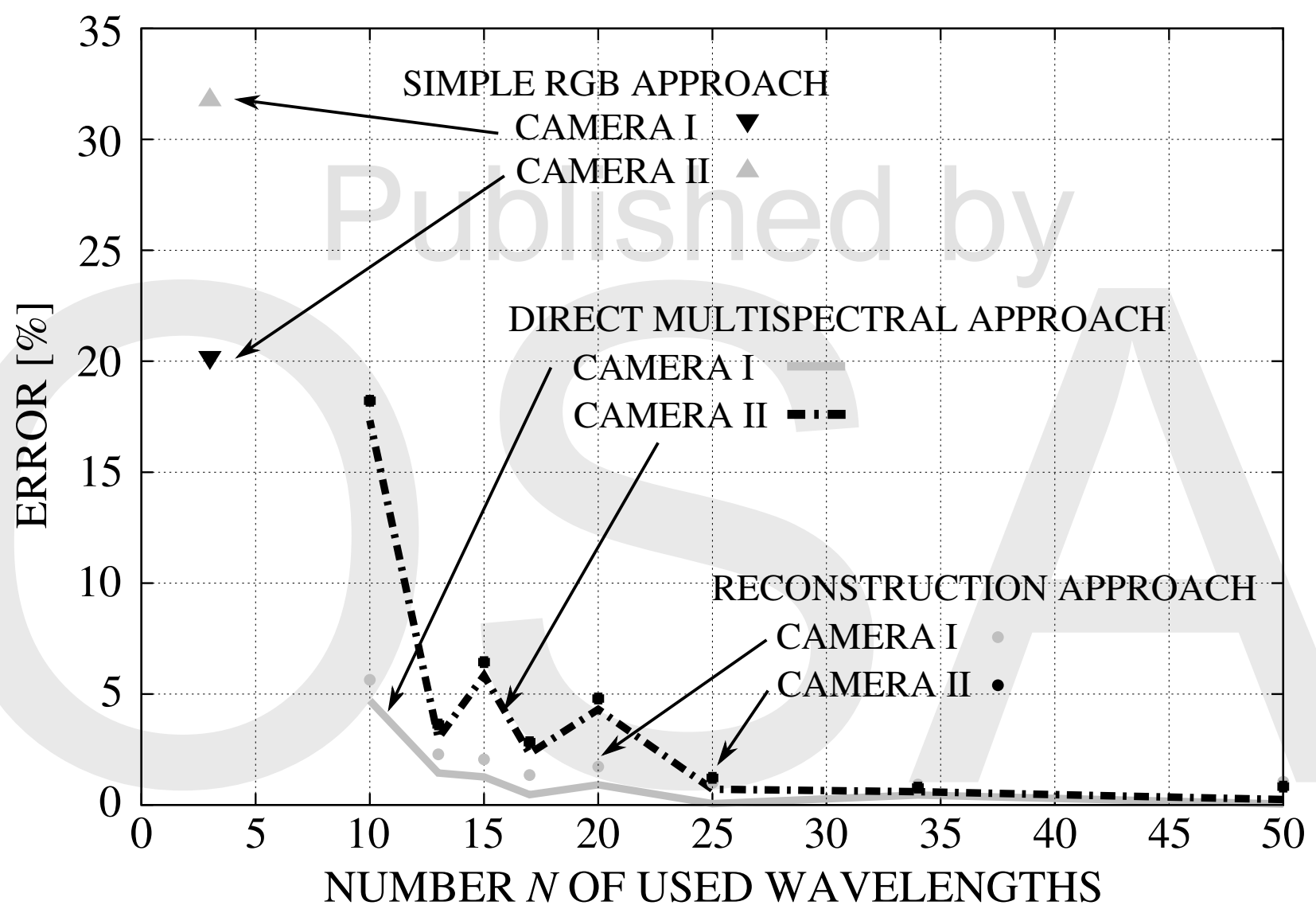

Fig. 8. Same as Fig. 7, but for type 2 water with $Z_{o}=7 \mathrm{~m}$. 
TYPE 1 WATER
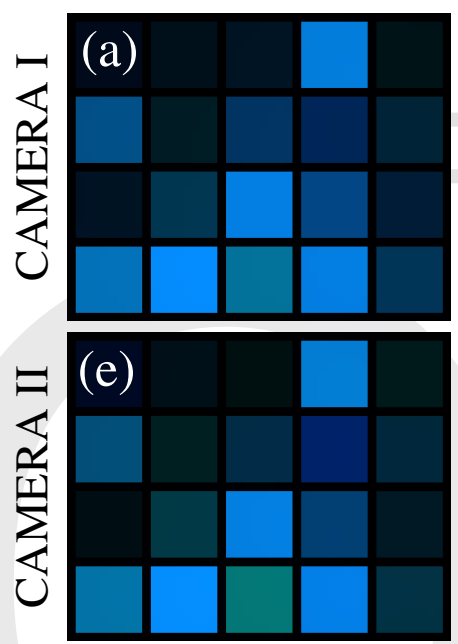

Simple RGB approach
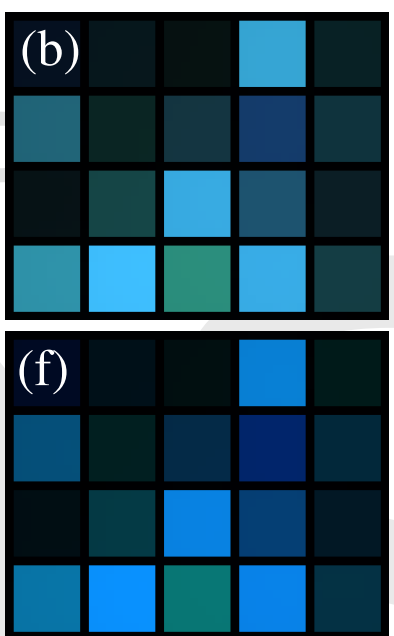

Reconstruction approach
TYPE 2 WATER
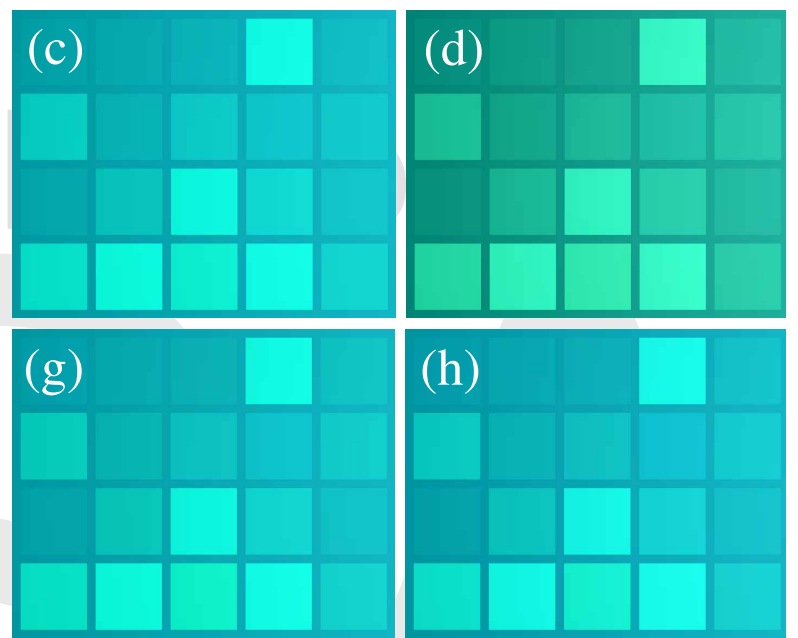

Simple RGB approach

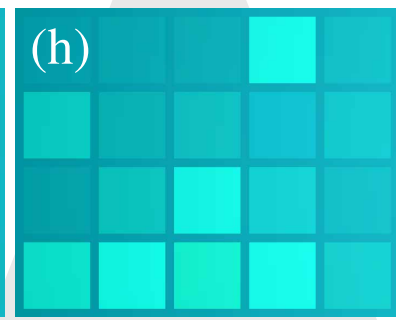

Reconstruction approach

Fig. 9. [color on-line] Visual aspect of scene of Fig.4 in subsea environments for $Z_{o}=7 \mathrm{~m}$. Upper row: The scene was imaged using camera I. Bottom row: Idem with camera II. Left columns: Results of simulation with for type 1 water. Right columns: Idem for type 2 water. (a), (c), (e), (g): Simulations were performed with the simple RGB approach. (b), (d), (f), (h): Idem with the reconstruction approach. 
(a) RED CHANNEL

(b) BLUE CHANNEL

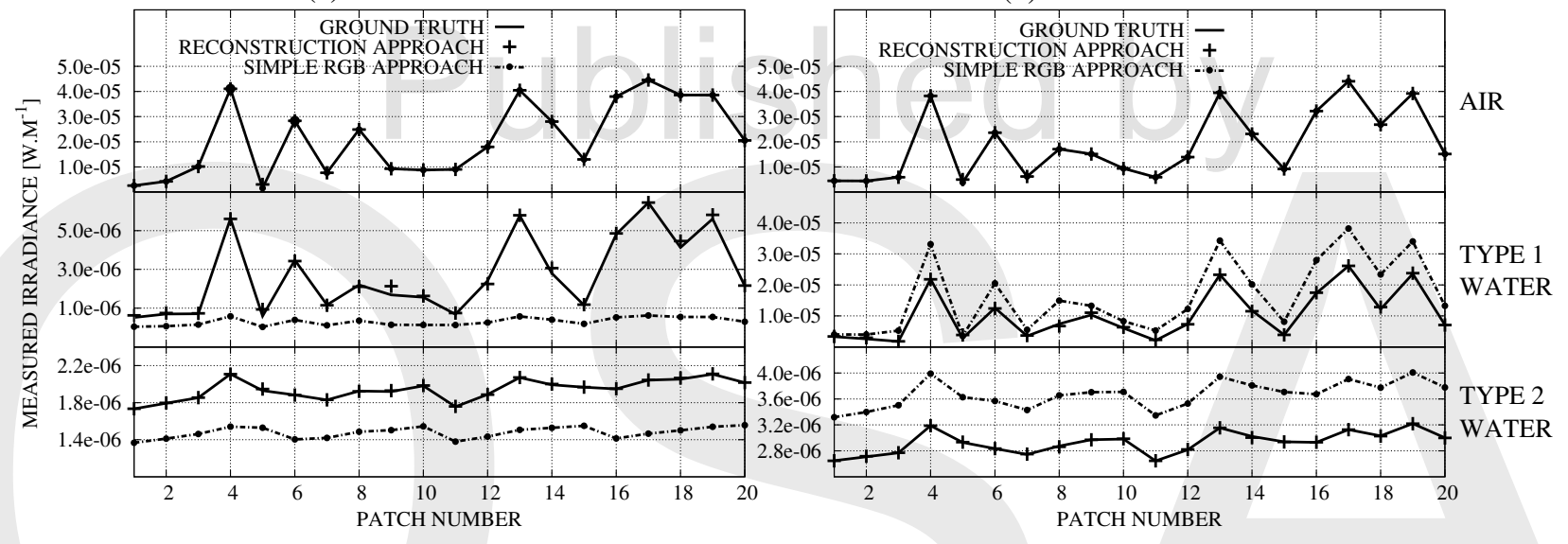

Fig. 10. Irradiance profiles of the 20 patches corresponding to the images of Fig. 4.(a) simulated for $Z_{o}=7 \mathrm{~m}$ with camera I. Row 1: Irradiance profiles in air. Row 2: Irradiance profiles in type 1 water. Row 3: Irradiance profiles in type 2 water. Plain curves: Ground truth. Dots and dashed line: Simple RGB approach. Crosses: Reconstruction approach. (a) Red channel. (b) Blue channel. 


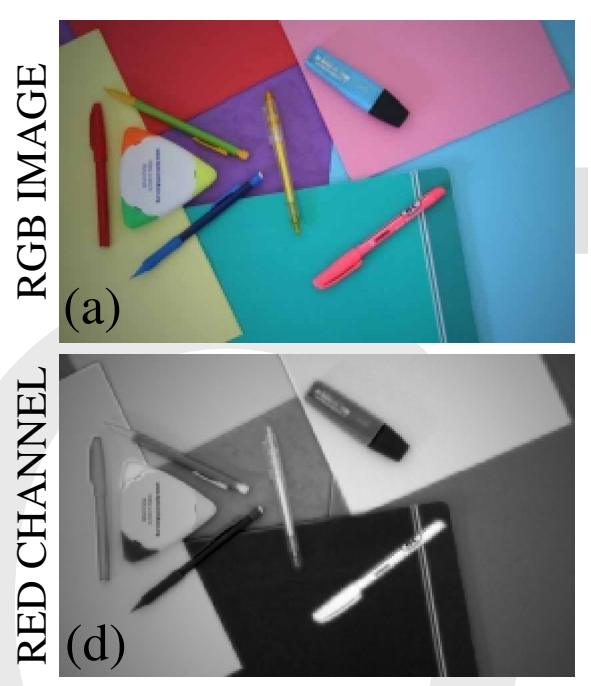

Initial image
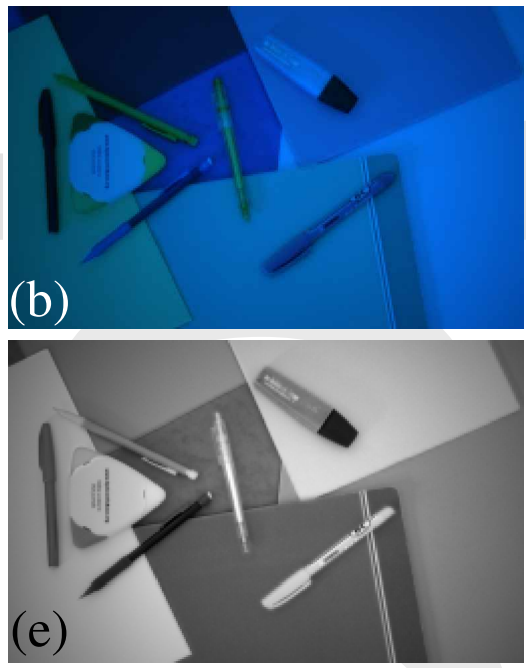

Reconstruction approach

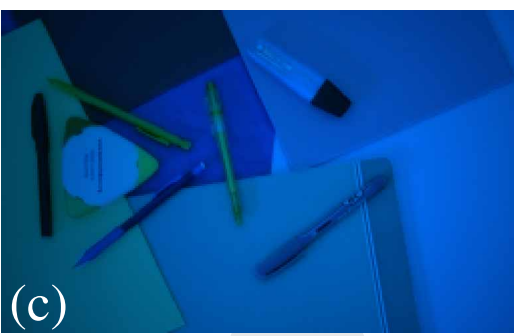

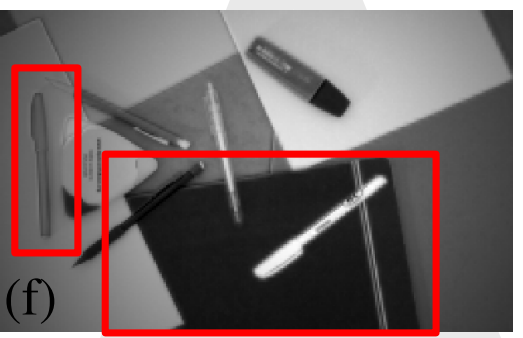

Simple RGB approach

Fig. 11. [color on-line] (a) Initial RGB image (256x160 pixels) taken with a standard camera. (b) Simulated RGB image for type 1 water and a distance $Z_{o}=7 \mathrm{~m}$ with a reconstruction step and the multispectral approach $(N=25)$. (c) Idem using the simple RGB approach. (d), (e), (f): Red channels correspond respectively to (a), (b) and (c). 